\title{
Prevalence of Anxiety and Depression in General Population of Iran during the COVID-19 Pandemic: A Web-Based Cross-Sectional Study
}

\section{Saman Maroufizadeh}

Guilan University of Medical Sciences

Majid Pourshaikhian

Guilan University of Medical Sciences

Ali Pourramzani

Guilan University of Medical Sciences

Farzaneh Sheikholeslami

Guilan University of Medical Sciences

Mohammad Taghi Moghadamnia

Guilan University of Medical Sciences

Seyed Amirhossein Alavi ( $\square$ alaviseyedamirhossein@gmail.com )

Guilan University of Medical Sciences https://orcid.org/0000-0002-4864-533X

Research

Keywords: Anxiety, Depression, COVID-19, General Population, Iran

Posted Date: June 30th, 2020

DOI: https://doi.org/10.21203/rs.3.rs-39082/v1

License: (9) (i) This work is licensed under a Creative Commons Attribution 4.0 International License.

Read Full License 


\section{Abstract}

Background: Coronavirus disease 2019 (COVID-19) is a global public health emergency and may result in psychological problems such as anxiety and depression in general population. Therefore, this study aimed to determine the prevalence of anxiety and depression and their associated factors in general population of Iran during the COVID-19 pandemic.

Methods: We conducted this web-based cross-sectional study on 5328 individuals in Iran, between 17 and 29 April 2020. Data were collected through an anonymous online questionnaire via social media like WhatsApp and Telegram. The online survey collected information on demographic characteristics, COVID-19-related variables, and anxiety and depression. Anxiety and depression were assessed using the Generalized Anxiety Disorder-7 (GAD-7) and the Patient Health Questionnaire-9 (PHQ-9), respectively. Simple and multiple logistic regression analyses were performed to determine independent predictors of anxiety and depression.

Results: The mean GAD-7 and PHQ-9 total scores were $7.17(\mathrm{SD}=5.42)$ and $7.80(\mathrm{SD}=6.68)$, respectively. The prevalence of anxiety, depression, and comorbid anxiety-depression were $30.1 \%, 33.4 \%$, and $22.1 \%$, respectively. According to the adjusted analysis, anxiety was significantly associated with being female, being young and middle-aged, being unemployed or housewife, having chronic diseases, long time thinking about COVID-19, having family members, friends, and/or relatives infected with COVI-19, and death of family members, relatives or friends due to COVID-19. Same results were also found for depression. Furthermore, depression was associated with being single, being resident in urban area, and having high risk individual in family.

Conclusion: The prevalence of anxiety and depression were considerably high in general population of Iran during the COVID-19 pandemic. In addition, the findings suggest that more attention needs to be paid to vulnerable groups such as women, young/middle-aged adults, unemployed people, people with chronic disease. Thus, a holistic approach, including management policies, psychosocial interventions, training, support, and tele-mental health, is absolutely crucial to reduce mental health problems of the COVID-19 and any outbreaks of other infectious diseases.

\section{Background}

The Coronavirus disease 2019 (COVID-19) is an infectious disease caused by severe acute respiratory syndrome coronavirus 2 (SARS-CoV-2). It was first identified in Wuhan, Hubei province, China, in December 2019. The World Health Organization declared the outbreak a Public Health Emergency of International Concern on January 30, 2020, and a pandemic on March 11, 2020. As of 27 May 2020, more than 5.61 million cases have been reported across 188 countries and territories, resulting in more than 350,000 deaths. The first confirmed case of COVID-19 in Iran was reported on February 19, 2020. As of May 27, 2020, 141,591 cases have been confirmed in Iran, based on the Ministry of Health and Medical Education report. There have been 7,564 deaths. 
As the COVID-19 pandemic rapidly sweeps across the world, it is leading to mental health problems such as of fear, stress, anxiety, depression, anger, and insomnia in the general population at large and particularly among special groups, such as older adults, healthcare workers, patients and people with underlying health conditions. Other factors that may increase the risk of developing these mental health problems include female sex, low socioeconomic status, frequent use of social media, low resilience and social support (1). One study of 1210 participants in China between January and February 2020 found that $53.8 \%$ of respondents rated the psychological impact of the COVID-19 pandemic as moderate or severe; $28.8 \%$ and $16.5 \%$ reported moderate to severe anxiety and depressive symptoms, respectively (1). Notwithstanding possible response bias, these are very high proportions-and it is likely that some people are at even greater risk. Beyond stresses inherent in the COVID-19 pandemic itself, related containment measures-including quarantine, social distancing, and self-isolation-can have a considerable effect on mental health and well-being. In particular, the increased loneliness and reduced social interactions are well-known risk factors for mental health problems such as anxiety and depressive disorders (2-4).

A growing body of evidence has also indicated that the outbreak of infectious diseases, including SARS, MERS, H1N1 and Ebola, were associated with poor mental health in general population $(5,6)$, healthcare workers $(7,8)$, patients and their family members (9). For example, in a study conducted during the SARS outbreak, symptoms of post-traumatic stress disorder and depression were observed in $28.9 \%$ and $31.2 \%$ of respondents, respectively (10).

The psychological impact of the COVID-19 pandemic must be acknowledged alongside the physical symptoms for general population, patients, and healthcare workers. On the other hand, early identification of persons at greater risk of psychological distress is essential in prevention and management strategies. Therefore, this study aimed to determine the prevalence of anxiety, depression, and comorbid anxietydepression in a large sample of the general population in Iran during the COVID-19 pandemic. We also examined the effect of demographic and COVID-19-related variables on these disorders.

\section{Methods}

\section{Participants and study design}

We performed this web-based cross-sectional study on 5328 individuals in Iran, between 17 and 29 April 2020. Data were collected through an anonymous online questionnaire via social media like WhatsApp and Telegram. The eligibility criteria for this study included (a) age over 18 years, (b) willingness to take part in the study, and (c) ability to read, write, and comprehend Persian.

\section{Ethical consideration}

This study was performed with the approval of the Ethics Committee of Guilan University of Medical Sciences, Rasht, Iran (Ethical Code: R.GUMS.REC.1399.020). All individuals were fully informed about the aim of the study and the voluntary nature of their participations. 


\section{Instruments}

Demographic variables

Demographic variables of participants including age, sex, marital status, parent status, place of residence, education, employment status, and ethnicity were collected.

COVID-19-related variables

The COVID-19-related variables included: (1) having chronic diseases, (2) times to focus on COVID-19 during a day, (3) contact with suspected or confirmed COVID-19 cases, (4) families, relatives or friends infected with COVID-19, (5) death of families, relatives or friends due to COVID-19.

Generalized Anxiety Disorder-7 (GAD-7)

The GAD-7 is a 7-item self-report scale that measures anxiety during the last two weeks based on DSM-IV criteria (11). Items are rated on a 4-point Likert-type scale ranging from 0 (not at all) to 3 (nearly every day). Total scores range from 0 to 21 , with higher scores indicating greater anxiety symptoms. A cut-off score of $\geq 10$ was used to identify individuals experiencing anxiety. This scale has been widely used in psychiatric, primary-care and general populations. The Persian version of this scale also has been reported to be a reliable and valid scale (12). In this study, the Cronbach's alpha coefficient of the GAD-7 was 0.871 .

Patient Health Questionnaire-9 (PHQ-9)

The PHQ-9 is a 9-item self-report scale that measures depression during the last two weeks based on DSM-IV criteria (13). Items are rated on a 4-point Likert-type scale ranging from 0 (not at all) to 3 (nearly every day). Total scores range from 0 to 27 , with higher scores indicating greater depressive symptoms. A cut-off score of $\geq 10$ was used to identify individuals experiencing depression. This scale has been widely used in psychiatric, primary-care and general populations. The Persian version of this scale also has been reported to be a reliable and valid scale (14). In this study, the Cronbach's alpha coefficient of the PHQ-9 was 0.894 .

\section{Statistical analysis}

In the present study, continuous variables were expressed as "mean (standard deviation (SD))" and categorical variables as "frequency (percentage)". Simple and multiple logistic regression analyses were undertaken to examine the association of anxiety and depression with demographic and COVID-19related variables. The crude and adjusted odds ratio (OR) and 95\% confidence interval (Cl) were calculated. Data analysis was undertaken using SPSS for windows, version 16.0 (SPSS Inc., Chicago, IL, USA). The level of significance was set at 0.05 , and all statistical tests were two-sided.

\section{Results}




\section{Participants characteristics}

The mean age of the participants was 32.95 ( $S D=10.53$ ) years (range: 18-90 years). Of the participants, $63.1 \%$ were female, 63.4 were young adults, $54.8 \%$ were married, $94.7 \%$ were resident in urban area (94.7\%), $76.1 \%$ had university education, $45.5 \%$ were employed, and $9.3 \%$ had chronic diseases. Other demographic and COVID-19-related characteristics of the participants are presented in Table 1.

Table 1. Demographic and COVID-19-related characteristics of the participants $(n=5328)$. 
n (\%)

\begin{tabular}{|lc}
\hline $\begin{array}{l}\text { Age (years) } \\
\text { Young adults (18-35) }\end{array}$ & $3377(63.4)$ \\
\hline Middle-aged adults (36-55) & $1775(33.3)$ \\
\hline Older adults (>56) & $176(3.3)$ \\
\hline Sex & $1966(36.9)$ \\
\hline Male & $3362(63.1)$ \\
\hline Female & \\
\hline Marital status & $2407(45.2)$ \\
\hline Single & $2921(54.8)$ \\
\hline Married & \\
\hline Place of residence & $5048(94.7)$ \\
\hline Urban & $280(5.3)$
\end{tabular}

\section{Education}

Primary

124 (2.3)

Secondary

1151 (21.6)

University

4053 (76.1)

\section{Occupation}

Employed

2424 (45.5)

Housewife

1040 (19.5)

Retired

180 (3.4)

Student

1161 (21.8)

Unemployed

$523(9.8)$

\section{Chronic diseases}

$\begin{array}{ll}\text { No } & 4830(90.7) \\ \text { Yes } & 498(9.3)\end{array}$

Times to focus on COVID-19 during a day

$<0.5 \mathrm{~h}$ 
Contact with suspected or confirmed COVID-19 cases

\begin{tabular}{ll} 
No & $3701(69.5)$ \\
\hline Yes & $1627(30.5)$ \\
\hline
\end{tabular}

Families, relatives or friends infected with COVID-19

\begin{tabular}{ll} 
No & $4575(85.9)$ \\
\hline Yes & $753(14.1)$ \\
\hline
\end{tabular}

Death of families, relatives or friends due to COVID-19

\begin{tabular}{cc}
\hline No & $4477(84.0)$ \\
\hline Yes & $851(16.0)$ \\
\hline High risk individual in family & \\
\hline No & $1781(33.4)$ \\
\hline Yes & $3547(66.6)$
\end{tabular}

COVID-19: Coronavirus Disease 2019.

\section{Distribution of GAD-7 and PHQ-9 scores}

The mean GAD-7 and PHQ-9 scores for all respondents were $7.17(\mathrm{SD}=5.42)$ and $7.80(\mathrm{SD}=6.68)$, respectively. Table 2 also presents the distribution of scores falling within GAD-7 and PHQ-9 severity cutoffs. Using a cut-off value of 10 for both measures, the prevalence of anxiety, depression, and comorbid anxiety-depression were $30.1 \%(n=1605), 33.4 \%(n=1779)$, and $22.1 \%(n=1178)$, respectively. Overall, females and young adults reported anxiety and depressive symptoms more frequently than males and older adults (see Table 2 and Table 3 ).

Table 2. Distribution of participants across the GAD-7 and PHQ-9 severity ratings $(n=5328)$ 


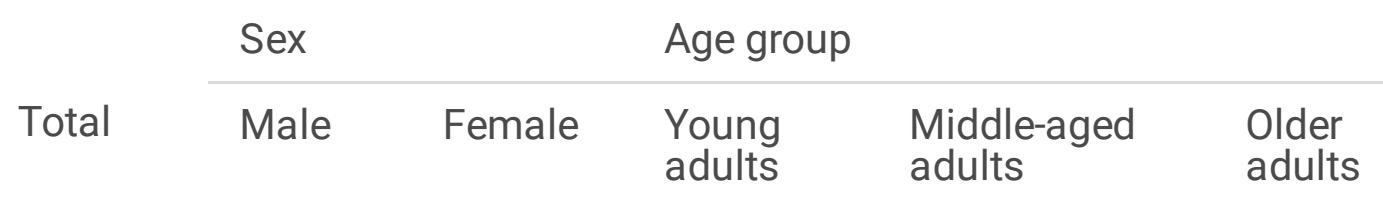

\begin{tabular}{|c|c|c|c|c|c|c|}
\hline No anxiety (0-4) & $\begin{array}{l}2033 \\
(38.2)\end{array}$ & $\begin{array}{l}863 \\
(43.9)\end{array}$ & $\begin{array}{l}1170 \\
(34.8)\end{array}$ & $\begin{array}{l}1255 \\
(37.2)\end{array}$ & $692(39.0)$ & $86(48.9)$ \\
\hline Mild (5-9) & $\begin{array}{l}1690 \\
(31.7)\end{array}$ & $\begin{array}{l}580 \\
(29.5)\end{array}$ & $\begin{array}{l}1110 \\
(33.0)\end{array}$ & $\begin{array}{l}1124 \\
(33.3)\end{array}$ & $516(29.1)$ & $50(28.4)$ \\
\hline Moderate (10-14) & $\begin{array}{l}962 \\
(18.1)\end{array}$ & $\begin{array}{l}307 \\
(15.6)\end{array}$ & $\begin{array}{l}655 \\
(19.5)\end{array}$ & $\begin{array}{l}606 \\
(17.9)\end{array}$ & $327(18.4)$ & 29 (16.5) \\
\hline Severe (15-21) & $\begin{array}{l}643 \\
(12.1)\end{array}$ & $\begin{array}{l}216 \\
(11.0)\end{array}$ & $\begin{array}{l}427 \\
(12.7)\end{array}$ & $\begin{array}{l}392 \\
(11.6)\end{array}$ & $240(13.5)$ & $11(6.2)$ \\
\hline \multicolumn{7}{|l|}{ PHQ-9 } \\
\hline None-minimal (0-4) & $\begin{array}{l}2124 \\
(39.9)\end{array}$ & $\begin{array}{l}887 \\
(45.1)\end{array}$ & $\begin{array}{l}1237 \\
(36.8)\end{array}$ & $\begin{array}{l}1232 \\
(36.5)\end{array}$ & 787 (44.3) & $\begin{array}{l}105 \\
(59.7)\end{array}$ \\
\hline Mild (5-9) & $\begin{array}{l}1425 \\
(26.7)\end{array}$ & $\begin{array}{l}487 \\
(24.8)\end{array}$ & $\begin{array}{l}938 \\
(27.9)\end{array}$ & $\begin{array}{l}902 \\
(26.7)\end{array}$ & $486(27.4)$ & $37(21.0)$ \\
\hline Moderate (10-14) & $\begin{array}{l}835 \\
(15.7)\end{array}$ & $\begin{array}{l}285 \\
(14.5)\end{array}$ & $\begin{array}{l}550 \\
(16.4)\end{array}$ & $\begin{array}{l}570 \\
(16.9)\end{array}$ & $249(14.0)$ & $16(9.1)$ \\
\hline $\begin{array}{l}\text { Moderately severe } \\
(15-19)\end{array}$ & $\begin{array}{l}563 \\
(10.6)\end{array}$ & $\begin{array}{l}163 \\
(8.3)\end{array}$ & $\begin{array}{l}400 \\
(11.9)\end{array}$ & $\begin{array}{l}400 \\
(11.8)\end{array}$ & $153(8.6)$ & $10(5.7)$ \\
\hline Severe (20-27) & $\begin{array}{l}381 \\
(7.2)\end{array}$ & $\begin{array}{l}144 \\
(7.3)\end{array}$ & $\begin{array}{l}237 \\
(7.0)\end{array}$ & $273(8.1)$ & $100(5.6)$ & $8(4.5)$ \\
\hline
\end{tabular}

COVID-19: Coronavirus Disease 2019; GAD-7: Generalized Anxiety Disorder-7; PHQ-9: Patient Health Questionnaire-9.

Values are presented as $\mathrm{n}(\%)$.

Table 3. Prevalence of anxiety, depression and comorbid anxiety-depression in general population in Iran during COVID-19 pandemic $(n=5328)$ 


\begin{tabular}{lllllll} 
& \multicolumn{3}{l}{ Sex } & \multicolumn{3}{c}{ Age group } \\
\cline { 3 - 7 } & Total & Male & Female & $\begin{array}{l}\text { Young } \\
\text { adults }\end{array}$ & $\begin{array}{l}\text { Middle-aged } \\
\text { adults }\end{array}$ & $\begin{array}{l}\text { Older } \\
\text { adults }\end{array}$ \\
\hline Anxiety & $\begin{array}{l}1605 \\
(30.1)\end{array}$ & $\begin{array}{l}523 \\
(26.6)\end{array}$ & $\begin{array}{l}1082 \\
(32.2)\end{array}$ & $998(29.6)$ & $567(31.9)$ & $40(22.7)$ \\
\hline Depression & $\begin{array}{l}1779 \\
(33.4)\end{array}$ & $\begin{array}{l}592 \\
(30.1)\end{array}$ & $\begin{array}{l}1187 \\
(35.3)\end{array}$ & $1243(36.8)$ & $502(28.3)$ & $34(19.3)$ \\
\hline Comorbidity & $\begin{array}{l}1178 \\
(22.1)\end{array}$ & $\begin{array}{l}384 \\
(19.5)\end{array}$ & $\begin{array}{l}794 \\
(23.6)\end{array}$ & $774(22.9)$ & $378(21.3)$ & $26(14.8)$ \\
\hline $\begin{array}{l}\text { Any } \\
\text { disorder }\end{array}$ & 2206 & 731 & 1475 & $1467(43.4)$ & $691(38.9)$ & $48(27.3)$ \\
\end{tabular}

COVID-19: Coronavirus Disease 2019.

Values are presented as $\mathrm{n}(\%)$.

\section{Factors associated with anxiety symptoms}

Simple and multiple logistic regression analyses were undertaken to examine factor associated with anxiety symptoms among general population (see Table 4). According to adjusted analysis (i.e., multiple logistic regression analysis), females were 1.20 times more likely to have anxiety than males (OR $\mathrm{Adj}=1.20,95 \% \mathrm{Cl}: 1.03-1.40)$. Young adults and middle-aged adults reported higher anxiety symptoms compared with older adults $\left(\mathrm{OR}_{\mathrm{Adj}}=1.69,95 \% \mathrm{Cl}\right.$ : 1.07-2.65, and $\mathrm{OR}_{\mathrm{Adj}}=1.67,95 \% \mathrm{Cl}$ : 1.07-2.60, respectively). Compared with employed participants, those who are unemployed and women who are housewife had more anxiety symptoms $\left(\mathrm{OR}_{\mathrm{Adj}}=1.68,95 \% \mathrm{Cl}: 1.33-2.12\right.$, and $\mathrm{OR}_{\mathrm{Adj}}=1.27,95 \% \mathrm{Cl}$ : 1.03 1.55 , respectively). Participants with chronic diseases were 1.68 times more likely to be anxious than other participants $\left(\mathrm{OR}_{\mathrm{Adj}}=1.68,95 \% \mathrm{Cl}: 1.35-2.08\right)$. The odds of anxiety increased with increasing times to focus on COVID-19 per a day. Those who had family members, friends, and/or relatives infected with COVID-19 were more likely to have anxiety symptoms ( $\mathrm{OR}_{\mathrm{Adj}}=1.49,95 \% \mathrm{Cl}$ : 1.21-1.82). Furthermore, those who reported death of families, relatives or friends due to COVID-19 were more likely to have anxiety symptoms (OR Adj $=1.26,95 \%$ Cl: $1.05-1.51)$.

Table 4. Association between GAD and demographic/COVID-19-related variables among participants $(n=5328)$. 
Variables

Prevalence, Simple logistic n (\%) regression

\section{OR Crude $\quad P$}

$(95 \% \mathrm{Cl})$
Multiple logistic regression

$\mathrm{OR}_{\text {Adj }} \quad P$

$(95 \% \mathrm{Cl})$

\section{Age}

Young adults

$998(29.6) \quad 1.43(0.99 \quad 0.053$

$-2.04)$

1.69

(1.07-

0.024

2.65)

Middle-aged adults

$\begin{array}{lll}567(31.9) & 1.60(1.11 & 0.012 \\ & -2.30) & \end{array}$

1.67

$(1.07-$

0.024

2.60)

Older adults

$40(22.7)$

1

1

Sex

Male

$523(26.6) \quad 1$

1

Female

1082

(32.2)

$1.31(1.16<0.001$

$-1.48)$

1.20

(1.03-

0.016

1.40)

\section{Marital status}

Single

685 (28.5) 1

Married

$920(31.5)$

$1.16(1.03 \quad 0.016$
$-1.30)$

1

0.94

(0.79-

0.503

1.12)

\section{Place of residence}

Urban

1540

(30.5)

$1.45(1.09 \quad 0.010$

1.20

(0.88-

0.256

1.63)

Rural

65 (23.2)

1

1

\section{Education}

Primary

Secondary

University

49 (39.5)

$347(30.1)$

1)

$0.66(0.45 \quad 0.033$

$-0.97)$

1209

(29.8)
$0.65(0.45 \quad 0.021$

$-0.94)$
1

0.79

(0.51 -

0.281

1.21)

0.73

(0.48-

1.11)

\section{Occupation}


Housewife

$371(35.7) \quad 1.36(1.17<0.001$

1.27

0.023

$-1.59)$

(1.03-

1.55)

Retired

$40(22.2)$

$0.70(0.49 \quad 0.056$

0.82

(0.53-

0.385

1.28)

Student

$290(25.0) \quad 0.82(0.70$

0.014

0.99

(0.80 -

0.892

1.21)

Unemployed

$203(38.8) \quad \begin{array}{ll}1.56(1.28 & <0.001 \\ -1.90) & \end{array}$

1.68

(1.33-

$<0.001$

2.12)

\section{Chronic diseases}

\begin{tabular}{|c|c|c|c|c|c|}
\hline No & $\begin{array}{l}1402 \\
(29.0)\end{array}$ & 1 & & 1 & \\
\hline Yes & $203(40.8)$ & $\begin{array}{l}1.68(1.39 \\
-2.03)\end{array}$ & $<0.001$ & $\begin{array}{l}1.68 \\
(1.35- \\
2.08)\end{array}$ & $<0.001$ \\
\hline
\end{tabular}

Times to focus on COVID-19 during a day

\begin{tabular}{|c|c|c|c|c|c|}
\hline$<0.5 \mathrm{~h}$ & 364 (15.7) & 1 & & 1 & \\
\hline $0.5-1 \mathrm{~h}$ & $244(22.4)$ & $\begin{array}{l}1.56(1.30 \\
-1.86)\end{array}$ & $<0.001$ & $\begin{array}{l}1.58 \\
(1.31- \\
1.89)\end{array}$ & $<0.001$ \\
\hline $1-2 \mathrm{~h}$ & 267 (36.7) & $\begin{array}{l}3.11(2.58 \\
-3.75)\end{array}$ & $<0.001$ & $\begin{array}{l}3.18 \\
(2.63- \\
3.85)\end{array}$ & $<0.001$ \\
\hline$>2 \mathrm{~h}$ & $730(61.2)$ & $\begin{array}{l}8.47(7.21 \\
-9.96)\end{array}$ & $<0.001$ & $\begin{array}{l}8.26 \\
(7.00- \\
9.73)\end{array}$ & $<0.001$ \\
\hline
\end{tabular}

\section{Contact with suspected or} confirmed COVID-19 cases

$\begin{array}{llllll}\text { No } & 1067 & 1 & & 1 & \\ & (28.8) & & & & \\ \text { Yes } & 538(33.1) & \begin{array}{l}1.22(1.08 \\ -1.38)\end{array} & 0.002 & 0.97 & 0.759 \\ & & & & (0.83- & \\ & & & & 1.15)\end{array}$

Families, relatives or friends infected with COVID-19

$\begin{array}{llllll}\text { No } & 1320 & 1 & & 1 & \\ & (28.9) & & & & \\ \text { Yes } & 285(37.8) & 1.50(1.28 & <0.001 & 1.49 & <0.001\end{array}$




\section{Death of families, relatives or friends due to COVID-19}

\begin{tabular}{clllll}
\hline No & $\begin{array}{l}1304 \\
(29.1)\end{array}$ & 1 & & 1 & \\
Yes & $301(35.4)$ & $\begin{array}{l}1.33(1.14 \\
-1.55)\end{array}$ & $<0.001$ & $\begin{array}{l}1.26 \\
(1.05-\end{array}$ & 0.012 \\
& & & $1.51)$ & \\
\hline High risk individual in family & & & & & \\
\hline No & $491(27.6)$ & 1 & & 1 & \\
Yes & 1114 & $1.20(1.06$ & 0.004 & 1.10 & 0.177 \\
& $(31.4)$ & $-1.36)$ & & $0.96-$ & \\
& & & & $1.27)$ & \\
\end{tabular}

\section{Factors associated with depressive symptoms}

Simple and multiple logistic regression analyses were undertaken to examine factor associated with depressive symptoms among general population (see Table 5). According to adjusted analysis, females were 1.18 times more likely to have depression than males $\left(\mathrm{OR}_{\mathrm{Adj}}=1.18,95 \% \mathrm{Cl}\right.$ : 1.03-1.36). Young adults and middle-aged adults reported higher depressive symptoms compared with older adults $\left(O \mathrm{R}_{\mathrm{Adj}}=2.03\right.$, 95\% Cl: 1.29-3.19, and OR ${ }_{\mathrm{Adj}}=1.57,95 \% \mathrm{Cl}$ : 1.00-2.44, respectively). Depression was less prevalent in married participants compared with those not married $\left(\mathrm{OR}_{\mathrm{Adj}}=0.77,95 \% \mathrm{Cl}: 0.66-0.91\right)$. Those who were resident in urban area reported higher depressive symptoms compared with those who were resident in rural areas ( $\left.\mathrm{OR}_{\mathrm{Adj}}=1.65,95 \% \mathrm{Cl}: 1.23-2.20\right)$. Compared with employed participants, those who are unemployed and women who are student had more depressive symptoms ( $\mathrm{OR}_{\mathrm{Adj}}=2.29,95 \% \mathrm{Cl}$ : 1.852.84, and $O R_{A d j}=1.42,95 \% \mathrm{Cl}: 1.18-1.71$, respectively). Participants with chronic diseases were 1.81 times more likely to be depressed than other participants $\left(\mathrm{OR}_{\mathrm{Adj}}=1.81,95 \% \mathrm{Cl}: 1.47-2.23\right)$. The odds of depression increased with increasing times to focus on COVID-19 per a day. Those who had family members, friends, and/or relatives infected with COVI-19 were more likely to have anxiety symptoms (OR $\mathrm{Adj}=1.30,95 \% \mathrm{Cl}: 1.07-1.57)$. The depression rate in those who reported death of families, relatives or friends due to COVID-19 was more than other participants ( $\mathrm{OR}_{\mathrm{Adj}}=1.17,95 \% \mathrm{Cl}$ : 0.99-1.39), although the difference was not statistically significant $(P=0.066)$. Participants who had high risk individual in their family were more likely to have depressive symptoms than other participants (OR ${ }_{\mathrm{Adj}}=1.24,95 \% \mathrm{Cl}$ : 1.09 1.41).

Table 5. Association between depression and demographic/COVID-19-related variables among participants $(n=5328)$. 
Variables

Prevalence, Simple logistic n (\%) regression

\section{OR Crude $\quad P$}

$(95 \% \mathrm{Cl})$
Multiple logistic regression

$\mathrm{OR}_{\text {Adj }} \quad P$

(95\% Cl)

\section{Age}

Young adults

1243

(36.8)

$502(28.3)$

Middle-aged adults

Older adults

Sex

Male

Female
$592(30.1) \quad 1$

1187

(35.3) $\begin{array}{ll}2.43(1.66< & <0.001 \\ -3.56) & \end{array}$

2.03

$(1.29-$

3.19)

1.57

$(1.00-$

2.44)

$34(19.3)$

1

1

\section{Marital status}

Single

Married

$\begin{array}{lll}927(38.5) & 1 & \\ 852(29.2) & 0.66(0.59 \quad<0.001 \\ & -0.74)\end{array}$

1

$\begin{array}{ll}0.77 & 0.001 \\ (0.66- & \\ 0.91) & \end{array}$

\section{Place of residence}

Urban

1711

(33.9)

$68(24.3)$

Rural

\section{Education}

Primary

Secondary

University

$42(33.9) \quad 1 \quad 1$

$\begin{array}{lllll}411(35.7) & 1.08(0.73 & 0.685 & 1.00 & 0.995 \\ & -1.60) & & (0.66- & \\ & & & 1.52) & \end{array}$

1326

(32.7)
$1.60(1.21<0.001$

-2.11 )
1.65

$(1.23-$

2.20)

0.002

0.048

$(1.03-$

0.019

1

$\begin{array}{ll}0.83 & 0.373 \\ (0.55- & \\ 1.25) & \end{array}$

\section{Occupation}


Housewife

$331(31.8) \quad 1.15(0.98 \quad 0.091$

$-1.34)$

1.10

$(0.90-$

0.359

1.33)

Retired

$31(17.2) \quad 0.51(0.34<0.001$

0.69

(0.44-

0.106

1.08)

Student

$452(38.9) \quad \begin{array}{ll}1.56(1.35 & <0.001 \\ -1.81) & \end{array}$

1.42

(1.18-

$<0.001$

Student

$263(50.3) \quad \begin{array}{ll}2.48(2.05 & <0.001\end{array}$

1.71)

Unemployed

2.29
$(1.85-$
$2.84)$

$<0.001$

\section{Chronic diseases}

\begin{tabular}{|c|c|c|c|c|c|}
\hline No & $\begin{array}{l}1571 \\
(32.5)\end{array}$ & 1 & & 1 & \\
\hline Yes & $208(41.8)$ & $\begin{array}{l}1.49(1.23 \\
-1.80)\end{array}$ & $<0.001$ & $\begin{array}{l}1.81 \\
(1.47- \\
2.23)\end{array}$ & $<0.001$ \\
\hline
\end{tabular}

Times to focus on COVID-19 during a day

\begin{tabular}{|c|c|c|c|c|c|}
\hline$<0.5 \mathrm{~h}$ & $576(24.8)$ & 1 & & 1 & \\
\hline $0.5-1 \mathrm{~h}$ & $323(29.7)$ & $\begin{array}{l}1.28(1.09 \\
-1.50)\end{array}$ & 0.003 & $\begin{array}{l}1.29 \\
(1.09- \\
1.52)\end{array}$ & 0.003 \\
\hline $1-2 \mathrm{~h}$ & 274 (37.6) & $\begin{array}{l}1.83(1.53 \\
-2.18)\end{array}$ & $<0.001$ & $\begin{array}{l}1.97 \\
(1.64- \\
2.37)\end{array}$ & $<0.001$ \\
\hline$>2 \mathrm{~h}$ & $606(50.8)$ & $\begin{array}{l}3.13(2.70 \\
-3.62)\end{array}$ & $<0.001$ & $\begin{array}{l}3.33 \\
(2.86- \\
3.89)\end{array}$ & $<0.001$ \\
\hline
\end{tabular}

\section{Contact with suspected or} confirmed COVID-19 cases

$\begin{array}{llllll}\text { No } & 1186 & 1 & & 1 & \\ & (32.0) & & & & \\ \text { Yes } & 593(36.4) & \begin{array}{l}1.22(1.08 \\ -1.37)\end{array} & 0.002 & 1.05 & 0.516 \\ & & & & 1.90- & \\ & & & & 1.22) & \end{array}$

Families, relatives or friends infected with COVID-19

$\begin{array}{llllll}\text { No } & 1487 & 1 & & 1 & \\ & (32.3) & & & & \\ \text { Yes } & 301(40.0) & 1.40(1.19 & <0.001 & 1.30 & 0.007\end{array}$




\section{Death of families, relatives or friends due to COVID-19}

\begin{tabular}{clllll}
\hline No & $\begin{array}{l}1464 \\
(32.7)\end{array}$ & 1 & & 1 & \\
Yes & $315(37.0)$ & $\begin{array}{l}1.21(1.04 \\
-1.41)\end{array}$ & 0.015 & $\begin{array}{l}1.17 \\
(0.99-\end{array}$ & 0.066 \\
& & & & $1.39)$ & \\
\hline High risk individual in family & & & & & \\
No & $531(29.8)$ & 1 & & 1 & \\
Yes & 1248 & $1.28(1.13$ & $<0.001$ & 1.24 & 0.001 \\
& $(35.2)$ & $-1.44)$ & & $1.09-$ & \\
\end{tabular}

Cl: Confidence Interval; OR: Odds Ratio

\section{Discussion}

The COVID-19 pandemic is having a profound effect on all aspects of society, including mental health and physical health. Despite this fact, adequate resources are typically not provided to prevent, control or reduce its effects on mental health. The present study is the first nationwide large-scale survey of mental health status in the general population of Iran during the COVID-19 pandemic. Similar to other web-based studies $(1,15-20)$, the majority of participants in this study were female $(63.1 \%)$, in the 18-35 years age group (63.4\%), resident in urban area (94.7\%), and had university education (76.1\%). In this study, using the GAD-7 and PHQ-9 instruments, the prevalence of anxiety, depression, and comorbid anxietydepression were $30.1 \%, 33.4 \%$ and $22.1 \%$, respectively. In all, our results indicated that the mental health status of people in Iran may have deteriorated compared to in the pre-COVID-19 period. Other studies during COVID-19 pandemic have also used this scale to assess mental health in the general population $(15-17,19)$, patients and health workers (21-23). In a study performed in Wuhan, China during the COVID19 pandemic, the prevalence of anxiety (using GAD-7), depression (using WHO-5), and comorbid anxietydepression were $22.6 \%, 48.3 \%$ and $19.4 \%$, respectively (17). In other study conducted in Wuhan and Shanghai, the prevalence of anxiety using GAD-7 were $32.7 \%$ and $20.4 \%$, respectively. In a study by Liu et al. conducted from 13 to 30 February 2020 in the Chinese general population, the prevalence of anxiety (using GAD-7 $\geq 10$ ) and depression (using PHQ-9 $\geq 10$ ) were $16.8 \%$ and $24.1 \%$, respectively (16).

As expected, the present study shows that females were 1.20 and 1.18 times more likely to have anxiety and depression symptoms than males, respectively. This finding is in line with the studies from the general population in which anxiety and depressive disorders are more prevalent in females than in males $(1,16,20)$. Contrary to our expectation, we found strong evidence that the mental health of young and middle-aged adults may be significantly worse than that of older adults during the COVID-19 pandemic. 
Given that older adults may be at higher risk for contracting the COVID-19 based on their underlying medical conditions, the state of mental health was expected to be relatively poor in this group. The unemployed working-age responders had strikingly poorer mental health (i.e., higher anxiety and depression symptoms) compared to the employed participants. These findings are consistent with the studies from the general population in which anxiety and depression disorders are more common in unemployed people than in employed people $(24,25)$. Furthermore, compared to the employed participants, students had higher depression symptoms, and housewife women had higher anxiety symptoms. This is in line with the population-based studies from China, in which depression is more prevalent in students $(1,16)$. Also, the findings by Liu et al. $(2020)$ showed the level of anxiety and depression significantly increased among college student in mainland china during the COVID-19 pandemic (26). Having chronic diseases increased the likelihood of having anxiety and depressive symptoms by 68 and 81 percent, respectively. These findings are consistent with previous studies $(1,16$, $17,20,27)$, that showed some groups of people may be more vulnerable to infection because of general health or some particular illness.

In addition to the above-mentioned factors, some people, such as those who had special groups in the family (including elderly, pregnant women, breastfeeding mothers, people with compromised immune function, and patients with disability) and those who had family members, friends, and/or relatives infected with COVID-19 were at increased risk for adverse psychological outcomes.

The present study has several strengths that should be highlighted, including (a) population-based nationwide setting, (b) the relatively large sample size, (c) evaluating both anxiety and depressive symptoms. There are also a few limitations that should be noted when interpreting the findings. Major limitations of this study come from the web-based nature of the study. Similar to other web-based studies, these limitations were low response rate, demographic biases, and variations in computer literacy and internet access. The other limitation was the cross-sectional nature of the present study, which precludes the ability to infer causality between demographic/COVID-19-related variables and anxiety and depressive symptoms. Finally, we did not have the data on some potentially important factors such as economic status of the family, and stability of family income during the COVID-19 pandemic, and being infected with COVID-19.

\section{Conclusion}

The prevalence of anxiety and depression were considerably high in general population of Iran during the COVID-19 pandemic. In addition, the findings suggest that more attention needs to be paid to vulnerable groups such as women, young/middle-aged adults, unemployed people, people with chronic disease. It is hoped this epidemiological study will provide a comprehensive picture of psychological distress in general population for policy markers not only in the COVID-19 pandemic but also in other infectious diseases that affect the mental health of the community. Moreover, research funders and researchers must deploy resources to understand the psychological and social, and economic effects of the COVID19 pandemic. 


\section{Abbreviations}

COVID-19: Coronavirus Disease 2019; GAD-7: Generalized Anxiety Disorder-7; PHQ-9: Patient Health Questionnaire-9; SD: Standard Deviation; OR: Odds Ratio; Cl: Confidence Interval.

\section{Declarations}

\section{Ethics approval and consent to participate}

This study was performed with the approval of the Ethics Committee of Guilan University of Medical Sciences, Rasht, Iran (Ethical Code: R.GUMS.REC.1399.020). All individuals were fully informed about the aim of the study and the voluntary nature of their participations.

\section{Consent for publication}

Not applicable.

\section{Availability of data and materials}

The datasets used and/or analyzed during the present study are available from the corresponding author on reasonable request.

\section{Competing interests}

The authors declare no conflict of interest.

\section{Funding}

This research received no specific grant from any funding agency in the public, commercial, or not-forprofit sectors.

\section{Authors' contributions}

Study concept and design: SM, MP, AP, FS, MTM, SAA.

Acquisition, analysis, or interpretation of data: SM, SAA.

Drafting of the manuscript: SM, SAA.

Critical revision of the manuscript for important intellectual content: SM, MP, AP, FS, MTM, SAA.

Statistical analysis: SM.

Obtained funding: Not Applicable.

Administrative, technical, or material support: SM, MP, MTM, SAA. 
Study supervision: SM.

\section{Acknowledgements}

We thank the people who participated in this study, and the Guilan University of Medical Sciences, Rasht, Iran for supporting this study.

\section{References}

1. World Health Organization. Coronavirus disease 2019 (COVID-19) situation reports-135. 3 June 2020. https://www.who.int/docs/default-source/coronaviruse/situation-reports/20200603-covid-19-sitrep135.pdf?sfvrsn=39972feb_2. Accessed 3 June 2020.

2. Wang C, Pan R, Wan X, Tan Y, Xu L, Ho SC, et al. Immediate Psychological Responses and Associated Factors during the Initial Stage of the 2019 Coronavirus Disease (COVID-19) Epidemic among the General Population in China. Int J Environ Res Public Health 2020; 17, 1729.

3. Zhou X, Snoswell CL, Harding LE, Bambling M, Edirippulige S, Bai X, et al. The role of telehealth in reducing the mental health burden from COVID-19. Telemed J E Health 2020; 26:377-379.

4. Smith EM. Ethnic minorities: Life stress, social support, and mental health issues. Couns Psychol 1985; 13:537-579.

5. Fiorillo A, Gorwood P. The consequences of the COVID-19 pandemic on mental health and implications for clinical practice. EurPsychiatry 2020; 63.

6. Cowling BJ, Ng DM, Ip DK, Liao Q, Lam WW, Wu JT, et al. Community psychological and behavioral responses through the first wave of the 2009 influenza A (H1N1) pandemic in Hong Kong. J Infect Dis 2010; 202:867-876.

7. $\mathrm{KO} \mathrm{CH}, \mathrm{YEN} \mathrm{CF}, \mathrm{YEN} J \mathrm{Y}$, YANG MJ. Psychosocial impact among the public of the severe acute respiratory syndrome epidemic in Taiwan. Psychiatry Clin Neurosci 2006; 60:397-403.

8. Lu Y-C, Shu B-C, Chang Y-Y. The mental health of hospital workers dealing with severe acute respiratory syndrome. Psychother Psychosom 2006; 75:370-375.

9. Bai Y, Lin C-C, Lin C-Y, Chen JY, Chue CM, Chou P. Survey of stress reactions among health care workers involved with the SARS outbreak. Psychiatr Serv 2004; 55:1055-1057.

10. Page LA, Seetharaman S, Suhail I, Wessely S, Pereira J, Rubin GJ. Using electronic patient records to assess the impact of swine flu (influenza H1N1) on mental health patients. J Ment Health 2011; 20:60-69.

11. Hawryluck L, Gold WL, Robinson S, Pogorski S, Galea S, Styra R. SARS control and psychological effects of quarantine, Toronto, Canada. Emerg Infect Dis 2004; 10:1206-1212.

12. Spitzer RL, Kroenke K, Williams JB, Löwe B. A brief measure for assessing generalized anxiety disorder: the GAD-7. Arch Intern Med 2006; 166:1092-1097.

13. Omani-Samani R, Maroufizadeh S, Ghaheri A, Navid B. Generalized Anxiety Disorder-7 (GAD-7) in people with infertility: A reliability and validity study. Middle EastFertil Soc J 2018. 
14. Kroenke K, Spitzer RL. The PHQ-9: a new depression diagnostic and severity measure. Psychiatr Ann 2002; 32:509-515.

15. Maroufizadeh S, Omani-Samani R, Almasi-Hashiani A, Amini P, Sepidarkish M. The reliability and validity of the Patient Health Questionnaire-9 (PHQ-9) and PHQ-2 in patients with infertility. Reprod Health 2019; 16:137.

16. Qian M, Wu Q, Wu P, Hou Z, Liang Y, Cowling J, et al. Psychological responses, behavioral changes and public perceptions during the early phase of the COVID-19 outbreak in China: a population based cross-sectional survey. MedRxiv 2020; 20024448.

17. Liu D, Ren Y, Yan F, Li Y, Xu X,Yu X, et al. Psychological Impact and Predisposing Factors of the Coronavirus Disease 2019 (COVID-19) Pandemic on General Public in China. SSRN Electronic Journal 2020.

18. Gao J, Zheng P, Jia Y, Chen H, Mao Y, Chen S, et al. Mental health problems and social media exposure during COVID-19 outbreak. PLoS One 2020; 15:e0231924-e.

19. Li J, Yang Z, Qiu H, Wang , Jian L, Ji J, et al. Anxiety and depression among general population in China at the peak of the COVID-19 epidemic. World Psychiatry 2020; 19:249-250.

20. Cao W, Fang Z, Guoqiang H, Han M, Xu X, Dong J, et al. The psychological impact of the COVID-19 epidemic on college students in China. Psychiatry Res 2020; 287:112934.

21. Mazza C, Ricci E, Biondi S, Colasanti M, Ferracuti S, Napoli C, et al. A Nationwide Survey of Psychological Distress among Italian People during the COVID-19 Pandemic: Immediate Psychological Responses and Associated Factors. Int J Environ Res Public Health 2020; 17:3165.

22. Lv, Yan and Yao, Huiqi and Xi, Yuandi and Zhang, Zhun and Zhang, Yuanqiu and Chen, et al. Social Support Protects Chinese Medical Staff from Suffering Psychological Symptoms in COVID-19 Defense. SSRN Electronic Journal 2020.

23. Lv, Yan and Zhang, Zhun and Zeng, Weijian and Li, Jie and Wang, Xuewen and Luo, et al. Anxiety and Depression Survey of Chinese Medical Staff Before and During COVID-19 Defense. SSRN Electronic Journal 2020.

24. Ying Y, Kong F, Zhu B, Ji Y, Lou Z, Ruan L. Mental health status among family members of health care workers in Ningbo, China during the Coronavirus Disease 2019 (COVID-19) outbreak: a Crosssectional Study. MedRxiv 2020; 20033290.

25. Watterson RA, Williams JV, Lavorato DH, Patten SB. Descriptive epidemiology of generalized anxiety disorder in Canada. Can J Psychiatry 2017; 62:24-29.

26. Kessler RC, Bromet EJ. The epidemiology of depression across cultures. Annu Rev Public Health 2013; 34:119-138.

27. Liu X, Liu J, Zhong X. Psychological State of College Students During COVID-19 Epidemic. SSRN Electronic Journal 2020.

28. Hu W, Su L, Qiao J, Zhu J, Zhou Y. Countrywide quarantine only mildly increased anxiety level during COVID-19 outbreak in China. MedRxiv 2020; 20041186. 